\title{
A Two-Gap Booster Synchrotron RF Cavity ${ }^{\dagger}$ W. R. Smythe and D. C. Van Westrum University of Colorado Boulder, CO 80309-0446
}

\section{Abstract}

Booster synchrotrons, such as the SSC Low Energy Booster, typically require a megavolt rf system which must tune about $25 \%$. The cavity proposed here is longitudinally symmetric with the gaps separated by $140^{\circ}$ in proton phase. The two perpendicularly biased ferrite tuners are directly connected to the center conductor and are located on either side of the cavity. The power tube is capacitively coupled to the midpoint of the drift tube. The cavity is designed to provide an energy gain of $1.88 \times 90 \mathrm{keV}$. The tuner features ferrite toroids which are bonded with epoxy to water cooled copper surfaces. The cavity walls are slotted so that a significant fraction of the ferrite can be tuned by high frequency components of the bias field.

\section{INTRODUCTION}

Since the first proposed application of transversely biased ferrite tuners to synchrotron of cavities [1],

†upported in part by the Superconducting Super Collider Laboratory, operated by Universities Research Association under contract with the U.S. DOE. there has been a continuing search for an optimum configuration. Most of the cavity configurations considered have been comprised of a single accelerating gap and a tuner which surrounds the beam line. Such tuners often have magnetic bias fields which result in a kilogauss size magnetic field in the beam line. An alternative configuration is proposed here, which is equivalent to two such cavities merged into a single cavity with two gaps, and which is distorted so that the tuners are not on the beam line. The cavity parameters have been chosen to match the requirements of the Low Energy Booster Synchrotron of the Superconducting Super Collider.

\section{Cavity Description}

The basic cavity geometry is illustrated in Figure 1. The gap spacing is $1.94 \mathrm{~m}$, which corresponds to $140^{\circ}$ in proton phase for the $5.00 \mathrm{~m}$ bunch spacing which is common to all of the SSC synchrotrons. The drift tube structure is supported in vacuum by the rf tuner windows, which are shown in Figure 2. The tuners are located outside of the accelerator vacuum and can be removed and replaced without breaking vacuum. There is good access to the drift tube through the center of

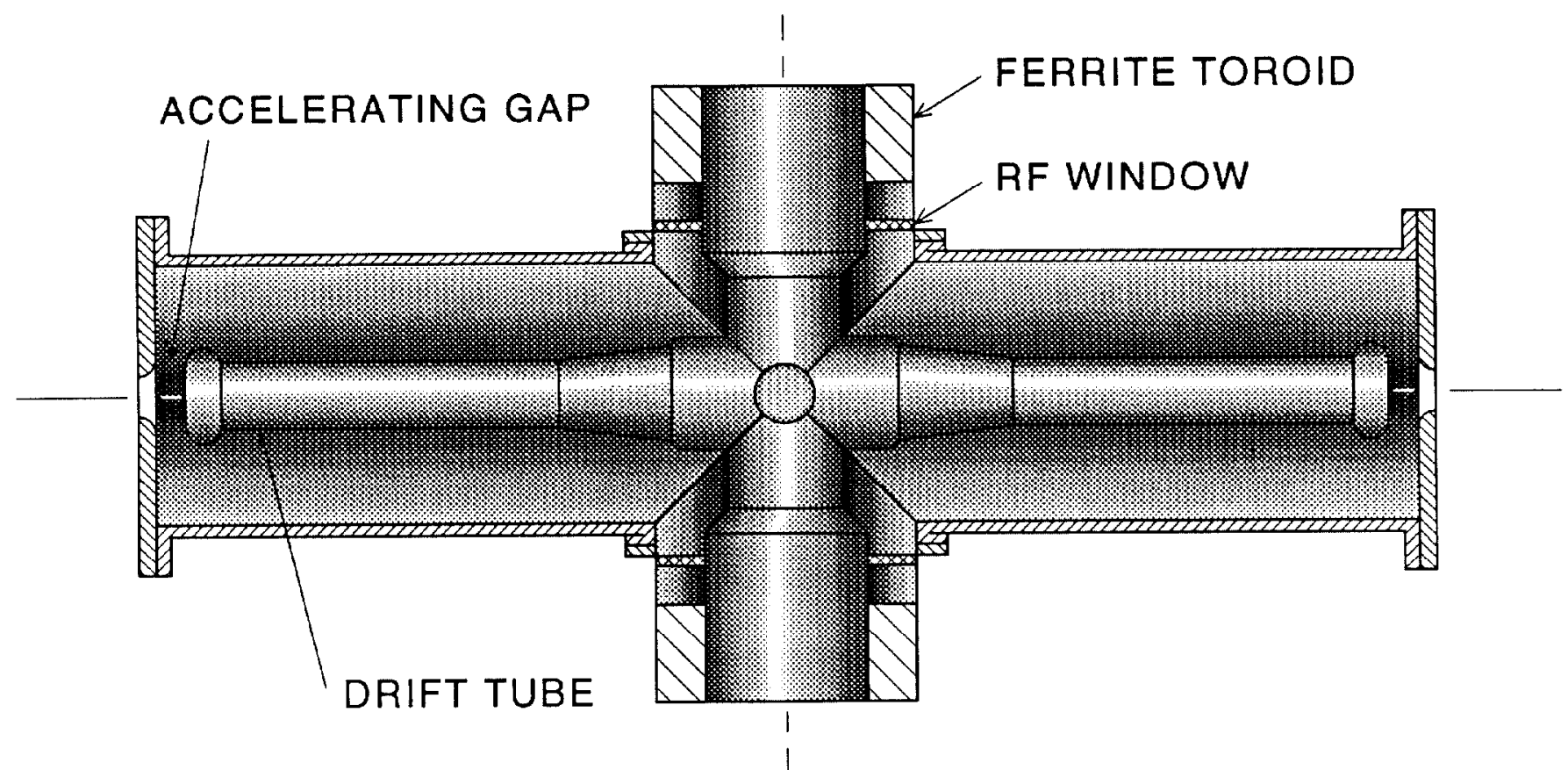

Figure 1. A partial horizontal cross section view of the cavity. The cavity consists of a long drift tube inside of a cylindrical tank with accelerating gaps at each end. The drift tube is supported by two coaxial ferrite tuners, as a cannon is supported by its trunnions. Not shown in this view is the power tube which sits verlically above the center of the drift tube and is capacitively coupled to it. 
the tuners for water cooling, alignment devices, probes, or even mechanical support. The power tube is mounted directly above the center of the cavity and is coupled to it by a cup-shaped, vacuum insulated capacitor. In addition, higher order mode dampers, previously described [2], would be added at each gap.

\section{TUNER DESIGN}

Each tuner consists of a ferrite filled short circuited coaxial line. As can be seen from Fig. 3 , it is very important that the bias field be quite uniform at the low frequency tuning limit, since the tuner dissipation rises very steeply with decreasing bias field. A laminated steel return yoke and coil configuration which achieves reasonable uniformity is illustrated in Fig. 4. The ferrite is bonded by epoxy to the water cooled copper surface which carries rf current around it, to cool the ferrite. The copper surface has longitudinal and radial slots extending around the ferrite. These slots inhibit eddy

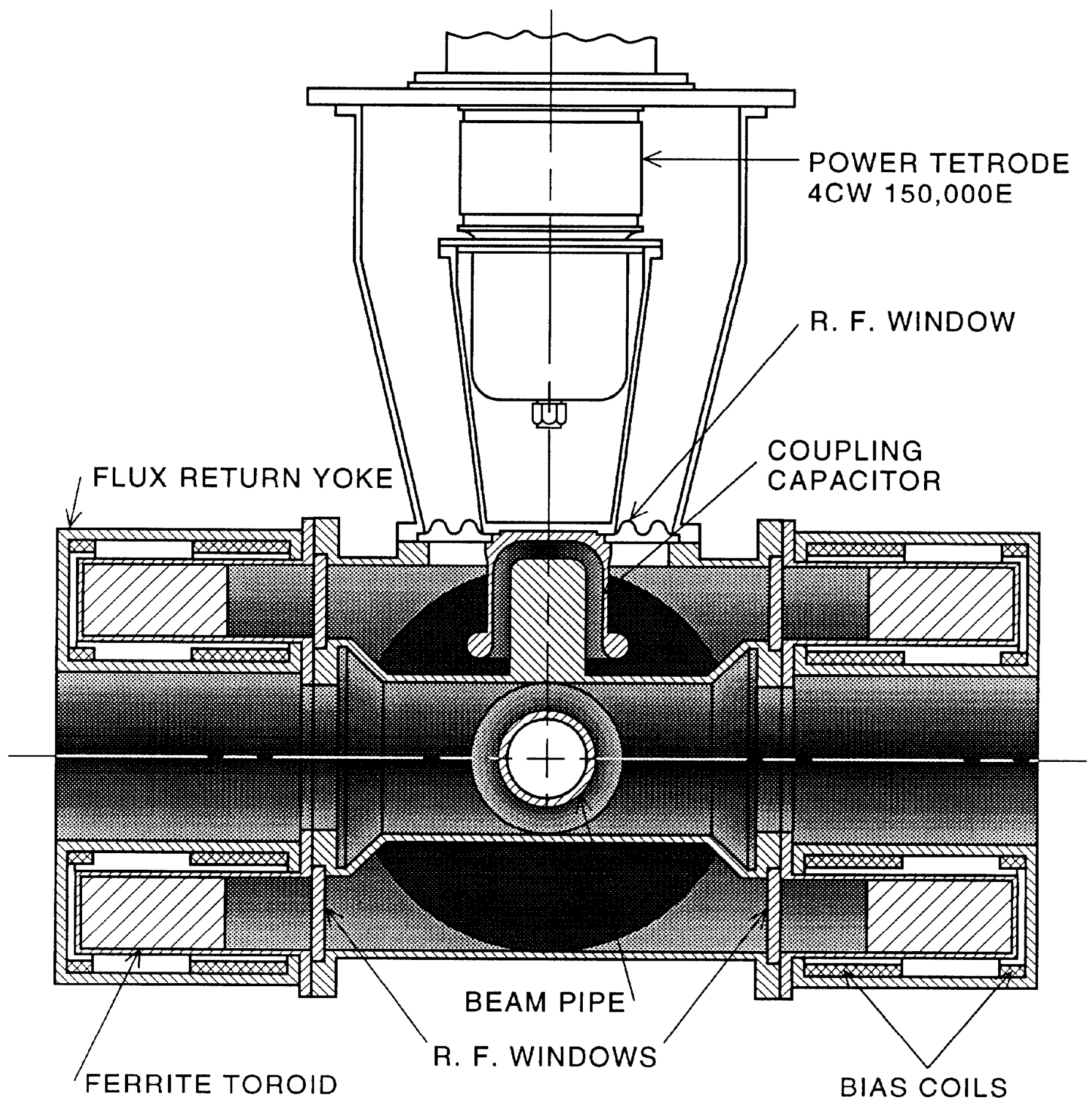

Figure 2. A vertical cross section through the center of the cavity, the power tube and the tuners, looking along the beam pipe. Each tuner can be removed and replaced without breaking the vacuum integrity. 


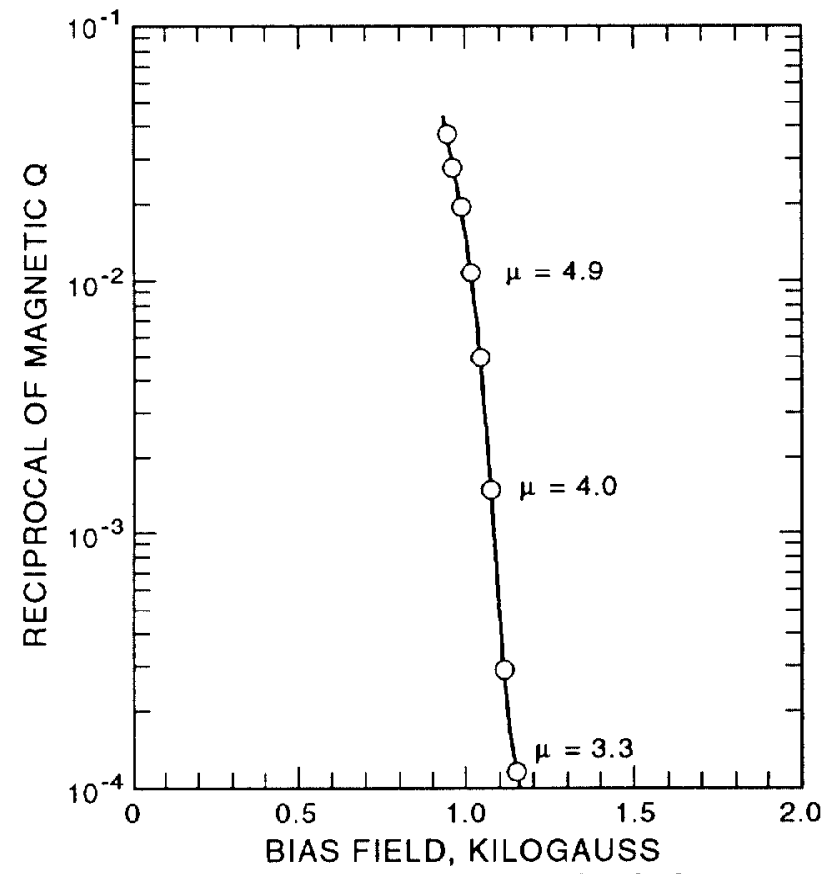

Figure 3. A plot of the reciprocal of the measured magnetic $Q$ of Trans-Tech G- 810 ferrite as a function of the dc magnetic bias field, for the case of perpendicular bias. The measurement method has been previously described [1]. The power dissipation in the ferrite is inversely proportional to the magnetic $Q$, so this plot makes clear the importance of achieving good bias field uniformity, when approaching the low bias field limit.

currents and permit high frequency components of the bias field to penetrate a large fraction of the ferrite volume, enhancing tuner frequency response.

\section{Analytichl Methods}

The primary method used to analyze this cavity has employed a transmission line model to calculate voltage distribution, currents, electric and magnetic energy density, power loss, etc. Approximations for gap capacitance, and conical shapes were verified by use of the computer program SUPERFISH. The approximation used for the four way coaxial intersection was validated by use of the three dimensional computer program MAFIA. The advantage of the transmission line method of analysis is the speed with which a variety of configurations can be explored.

\section{SUMmaRY AND DISCUSSION}

The calculated parameters of the cavity are listed in Table 1. It is seen that the cavity achieves the desired tuning range with reasonable power dissipation in the ferrite. This cavity employs about half as much ferrite per gap as do comparable single gap cavities [1], due to its lower stored energy. The lower stored energy results in a somewhat higher $\mathbf{R} / \mathrm{Q}$ per gap. A major mechanical simplification results from having a configuration in which the beam does not pass through the tuner. Tuner maintenance and replacement can be achieved while the cavity remains under vacuum.

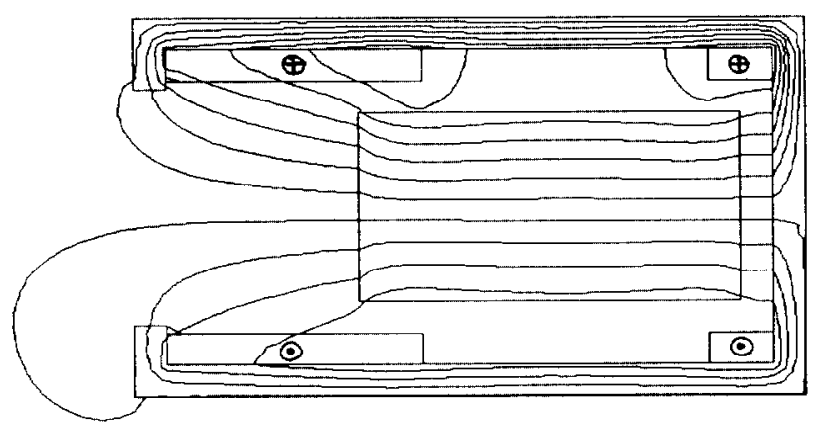

TUNER AXIS

Figure 4. Magnetic bias field plot at the low frequency limit, calculated with POISSON. Each inner coil current is equal and opposite to the corresponding outer coil current, resulting in zero net ampere turns about the tuner axis, and greatly reducing magnetic fields external to the tuner.

Table 1. Calculated Performance Characteristics of the Proposed 'Iwo-gap Cavity Design.

Frequency

Ferrite $\mu$

Gap voltage, each gap

Tuner/window voltage

Copper loss

$\mathrm{Q}$

R/Q (Two Gaps)

Ferrite

Ferrite bias $H$

Ferrite magnetic $Q$

Ferrite electric Q

Ave. ferrite pwr. dens.

Ferrite volume

Cavity length

Gap to gap beam phase

Energy gain

\section{ACKNOWLEDGEMENT}

We wish to express our appreciation to Linda Walling of the SSC Laboratory who kindly used the three-dimensional program MAFIA to provide runs for verification of the transmission line approximation to the "four-way junction" at the center of the cavity.

\section{REFERENCES}

[1] W.R.Smythe, T.G.Brophy, R.D.Carlini, C.C.Friedrichs, D.L.Grisham, G.Spalek, and L.C.Wilkerson, "RF Cavities with Transversely Biased Ferrite Tuning," IEEE Trans. Nucl. Sci. NS-32, No. 5, 2951 (1985).

[2] W.R.Smythe, C.C.Friedrichs, and L.S.Walling, "Proton Synchrotron RF Cavity Mode Damper Tests," Conference Record of the 1991 IEEE Particle Accelerator Conference, 643 (1991). 